\title{
THE EFFECTS OF VITAMIN D3 AND CALCIUM SUPPLEMENTATION ON FASTING PLASMA GLUCOSE, SERUM LIPIDS, RATE PRESSURE PRODUCT AND BODY MASS INDEX IN HEALTHY ADULTS
}

\author{
Aml Mohamed Nada \\ Department of Internal Medicine, Unit of Endocrinology, Diabetes and Metabolism, Faculty of Medicine, \\ Mansoura University, Mansoura 35516, Egypt.
}

\begin{abstract}
Aim: to investigate the effect of vitamin D3 replacement on body mass index, blood pressure, pulse rate, fasting plasma glucose and serum lipids, in healthy vitamin $\mathrm{D}$ deficient adults.

Patients and methods: Twenty-five healthy adult participants (3 males and 22 females) with vitamin D3 deficiency were recruited from the endocrine clinic in a tertiary care hospital in Saudi Arabia. History was taken and clinical examination was done for all participants. Height, weight, sitting blood pressure, and pulse rate were measured. Laboratory investigations in the form of vitamin D3 level, fasting plasma glucose, and lipid profile were measured before the participants received any oral vitamin D3. The same anthropometric and biochemical parameters were measured 9 months after starting vitamin D3 supplementation in the form of oral cholecalciferol. Replacement was given as 4500 units of vitamin D3 drops daily for one month, then 3000 units daily for another month, then 2000 units daily for seven months. This was combined with oral calcium carbonate intake of 500 milligrams daily throughout the nine month study period.

Results: Pretreatment serum vitamin D3 level was $9.01 \pm 3.2$ nanogram/milliliter. A vitamin D3 level of $35.4 \pm 6.84$ nanogram/milliliter was achieved by the end of the study. A significant reduction in the pulse rate $(p=0.000)$ and the systolic blood pressure was noticed $(p=0.002)$. The diastolic blood pressure did not significantly change $(p=0.148)$. The rate pressure product (RPP) was significantly reduced by the end of the study $(p<0.001)$. The increase in vitamin D3 level was not associated with significant changes in fasting plasma glucose $(p=0.184)$ or any of the measured components of the lipid profile. There was no significant effect on the body mass index $(p=0.31)$.

Conclusion: Vitamin D3 replacement is associated with a significant reduction of the pulse rate, the systolic blood pressure and the rate pressure product. This indicates a reduction of the cardiac work which may partially explain the cardioprotective effect of vitamin D3. The body mass index does not significantly change with vitamin D replacement . No significant effect of vitamin D replacement on fasting plasma glucose in healthy adults, who are neither diabetic nor prediabetic. Also, no effect of vitamin D replacement on the lipid profile in the same group of healthy population.
\end{abstract}

\section{INTRODUCTION}

$\mathrm{C}$ ardiovascular diseases (CVD) remain the leading cause of death and disability in the world. Thus, extraordinary effort has been devoted to determining the modifiable risk factors to prevent such diseases ${ }^{[1]}$.

In recent years, adequate vitamin D levels are demonstrated to be very important for optimal functioning of many organs and tissues throughout the body. Low vitamin D levels are found in association with diabetes mellitus, hypertension, metabolic syndrome, dyslipidemia, obesity, left ventricular hypertrophy, coronary artery calcification and chronic vascular inflammation $\left[{ }^{2-7]}\right.$.

Deficiency of vitamin D was shown to result in an increased expression of genes that are associated with activation of the renin-angiotensin system and genes that are associated with malfunction of calcium channels. Therefore, activation of these genes is likely to lead to hypertension, left ventricular hypertrophy and congestive heart failure ${ }^{[8]}$.
Hypovitaminosis D was reported as a risk factor for increased cardiovascular events among 1739 adult participants in the Framingham Offspring Study. Moreover, analysis of more than 13,000 adults in the Third National Health and Nutrition Examination Survey (NHANES III) showed that even though hypovitaminosis D is associated with an increased prevalence of CVD risk factors, its association with all-cause mortality is independent of these risk factors ${ }^{[9]}$.

More than 20 cross-sectional studies have examined the association between plasma 25(OH)D and either blood pressure or prevalent hypertension. The great majority of these studies demonstrate that lower circulating $25(\mathrm{OH}) \mathrm{D}$ levels are associated with higher blood pressures or a higher prevalence of hypertension, including large population-based cohorts in the United States, Germany, and the United Kingdom ${ }^{[10,11]}$. Type 2 diabetes mellitus is also associated with considerable morbidity and mortality being a major risk factor for CVD. Its prevalence has been increasing nationally and worldwide ${ }^{[12-14]}$. 
However, although weight-loss has been shown to be successful in delaying the onset of type 2 diabetes, it is difficult to achieve and maintain long-term [15].

Therefore, identification of weightindependent, easily modifiable risk factors is urgently needed to attenuate the increase in the incidence of type 2 diabetes. As there is an accumulating evidence that vitamin D insufficiency is associated with the risk of developing T2DM, studying the effect of replacement of such deficiency and its effect on glucose homeostasis is a point of recent interest [16-19].

This role of vitamin D is suggested by crosssectional studies showing that low serum $25(\mathrm{OH}) \mathrm{D}$ concentration is associated with overweight, obesity, glucose intolerance, diabetes, insulin resistance and the metabolic syndrome ${ }^{[20-}$ 22]. It was also reported that higher levels of $25(\mathrm{OH}) \mathrm{D}$ are associated with lower rates of development of diabetes ${ }^{[23-26]}$.

While observational studies generally support an inverse relation between serum vitamin $\mathrm{D}$ levels and the risk of T2DM, various interventional studies that had been conducted to date provided inconclusive results showing either no effect of vitamin D supplementation on the risk of developing diabetes [27] or an attenuation of the increase in glycemia and insulin resistance that occur over time ${ }^{[28]}$.

Obesity is a well known risk factor for T2DM as well as CVD. Several Cross-sectional studies have demonstrated an inverse relation between vitamin $\mathrm{D}$ levels in the blood and the body mass index, and that obese people have lower serum vitamin D3 levels. It is not clear till now which is the cause and which is the result ${ }^{[29,30]}$.

The relation between vitamin $\mathrm{D}$ and lipids has so far received little attention. A PubMed search identified 22 cross-sectional studies including a minimum of 500 subjects and a 10 placebocontrolled double-blind intervention studies with vitamin $\mathrm{D}$ where more than 50 subjects were included. In all the cross-sectional studies serum $25(\mathrm{OH}) \mathrm{D}$ was positively associated with highdensity lipoprotein cholesterol (HDL-C) resulting in a favourable low-density lipoprotein cholesterol (LDL-C) or total cholesterol to HDL-C ratio. There was also a uniform agreement between studies on a negative relation between serum 25(OH)D and triglycerides (TG). On the other hand, the intervention studies gave divergent results, with some showing a positive and some showing a negative effect of vitamin D supplementation ${ }^{[31]}$.
Some reports demonstrated that low vitamin D level is associated with high blood levels of inflammatory factors, including C-reactive protein and cytokines such as interleukin-10. These cytokines have recently been identified as having an important role in atherogenesis ${ }^{[32,5]}$. Vitamin D analogues have been shown to inhibit the production of these cytokines while stimulating the effects of helper T lymphocytes, thus reducing plaque production or instability ${ }^{[33]}$. This may indicate that the role of vitamin D deficiency in development of atherosclerosis is dependent on its effect on serum lipids.

Thirty to fifty percent of both children and adults in the United States, Canada, Europe, Australia, New Zealand, and Asia are vitamin D-deficient. Despite the important role of sunlight in vitamin D synthesis, recent studies have shown that the rate of vitamin D deficiency is also higher in the sunniest areas of the world, including the Middle East countries, such as Saudi Arabia, Qatar, and United Arab Emirates and Iran because of low exposure to sun due to cultural factors ${ }^{[34-37]}$.

Taking into account that vitamin D deficiency is highly prevalent across the world while vitamin D supplementation is simple, safe, and inexpensive, the deficiency of vitamin D may be a common and easily treatable risk factor for diabetes mellitus, dyslipidemia and hypertension and consequently for CVD prevention [38-40] .

The objective of the present study was to investigate the effect of vitamin D supplementation on the blood pressure, pulse rate, rate pressure product, BMI, fasting plasma glucose and the lipid profile, all of which play a significant role in the development of cardiovascular diseases.

\section{MATERIALS AND METHODS}

Twenty-five healthy adults ( 3 males and 22 females) in the age range of 18 to 50 years were recruited from the endocrine clinic in a tertiary care hospital in southern region of Saudi Arabia. All were deficient in vitamin D3. None of them has history of diabetes mellitus, impaired fasting plasma glucose, dyslipidaemia or hypertension. Exclusion criteria included intake of vitamin D or any other medication that may affect any of the measured variables, known history of hypercalcaemia, evidence of thyroid, renal, hepatic or cardiac disease, active sarcoidosis, tuberculosis or malignancy. Among women, current breast feeding, pregnancy or planning a pregnancy as well as menopause were also considered as exclusion criteria.

The study was approved by the Hospital Ethical Committee and written and informed consents 
were obtained from all participants. Height in meters (m), weight in killograms (kg) were measured using a stadiometer and a balance scale, respectively. Body mass index was calculated as weight in killograms divided by square the height in meters $(\mathrm{kg} / \mathrm{m} 2)$. Blood pressure was measured in seated subjects after a rest for at least 15 minutes using a mercury tensiometer. Radial pulse rate per minute was also taken with emphasis on regularity and equality on both sides. Rate pressure product was calculated as HR x SBP/100

Blood samples were collected after an overnight fasting. Vitamin D3 was assayed by Cobas e 601 \& Elecsys 2010 using liquid chromato-graghy tandem Mass Spectrometry ${ }^{[42]}$. Triglycerides, total cholesterol, LDL-C, HDL-C were measured using enzymatic colorimetric method on the Siemens Dimension RXL clinical chemistry system ${ }^{[43,44]}$. Fasting plasma glucose was determined on Siemens Dimension RXL chemistry analyzer using bichromatic (340 and $383 \mathrm{~nm}$ ) endpoint technique ${ }^{[45]}$.

Reference ranges; total cholesterol: 50-200 milligram per deciliter (mg/dl), triglycerides: 30-150 mg/dl, HDL-C: 35-55mg/dl, LDL-C: 100130mg/dl, Vitamin D3: 11-42 ng/ml.

Vitamin D deficiency was considered if the level was less than $20 \mathrm{ng} / \mathrm{ml}^{[46]}$. Subjects were started on replacement in the form of oral vitamin D 3 in a dose of 4500 units daily for one month then 3000 units daily for another month then 2000 units daily till the end of the study. All participants received calcium carbonate tablets in a dose of $500 \mathrm{mg}$ daily throughout the study period.

Anthropometric and biochemical parameters were assessed before and nine months after starting vitamin D and calcium supplements. Anthropometric parameters were measured by the same person and the same equipment on both occasions.

Statistical analysis: Data were entered into a computer using the statistical package for social sciences (SPSS) version 21.0. Descriptive statistics for quantitative variables were expressed in their means and standard deviations. values before and after intervention were compared using the paired t-test. Differneces were considered statistically significant when p-values were less than 0.05 .

\section{RESULTS}

The study showed a significant increase in vitamin D3 level after treatment $(9.01 \pm 3.2$ versus $35.4 \pm 6.84 \mathrm{ng} / \mathrm{ml}, p$-value $=0.000)$. There was a significant decrease in systolic blood pressure after replacement $(127.88 \pm 12.1 \quad$ versus $120.44 \pm 11.66$ respectively, $p$-value $=0.002)$. The mean decrease in SBP was $7.44 \mathrm{mmHg}$ with an average of $2.5 \%$ reduction of the pretreatment level. Pulse rate also significantly decreased [82.56 \pm 11.78 versus $74.88 \pm 7.83$ beat per minute $(\mathrm{bpm}), \quad p$-value $=0.000 \mathrm{]}$. The mean decrease in pulse rate was $7.68 \mathrm{bpm}$ with an average reduction of $8.2 \%$ of baseline pulse rate. No significant change in diastolic blood pressure was noticed. The rate pressure product significantly decreased by the end of the study $(105.32 \pm 16.2$ versus $90.0 \pm 11.4, p$-value $<0.001)$. See tables 1,2 Body mass index did not significantly change $(p=0.31)$. We did not find a significant effect of vitamin D3 replacement on fasting plasma glucose, triglycerides, total cholesterol, LDL-C or HDL-C. See tables 1, 2

Table(1) Comparison between different variables before and after vitamin $D$ replenishment

\begin{tabular}{llll}
\hline \multicolumn{5}{c}{ Before } & After & p-value \\
\hline Variable & Mean \pm SD & Mean \pm SD & .310 \\
\hline BMI & $32.62 \pm 7.42$ & $33 \pm 7.37$ & .250 \\
\hline wt & $77.68 \pm 18.82$ & $78.68 \pm 18.50$ & .000 \\
\hline P & $82.56 \pm 11.78$ & $74.88 \pm 7.83$ & .148 \\
\hline DBP & $78.12 \pm 10.72$ & $75.64 \pm 8.99$ & .002 \\
\hline SBP & $127.88 \pm 12.08$ & $120.44 \pm 11.6622$ & $<0.001$ \\
\hline RPP & $105.32 \pm 16.2$ & $90.02 \pm 11.4$ & .184 \\
\hline FPG & $97.48 \pm 14.96$ & $93.92 \pm 16.00$ & .395 \\
\hline TG & $105 \pm 71.17$ & $98.08 \pm 41.7751$ & .290 \\
\hline HDL-C & $41.85 \pm 10.66$ & $43.24 \pm 9.97$ & .311 \\
\hline LDL-C & $122.68 \pm 32.96$ & $114.98 \pm 44.42$ & .085 \\
\hline Tch & $187.16 \pm 33.71$ & $180.12 \pm 37.81$ & .000 \\
\hline D3 & $9.02 \pm 3.18$ & $35.40 \pm 6.84$ & \\
\hline
\end{tabular}


BMI:body mass index, wt:weight, P:pulse, DBP:diastolic blood pressure, SBP:systolic blood pressure, RPP: rate pressure product, FPG: fasting plasma glucose, TG: triglycerides, HDL-C:high density lipoprotein, LDL-C: low density lipoprotein, Tch: total cholesterol,

Table (2) Changes in different variables after vitamin D replenishment

\begin{tabular}{lll}
\hline Variable & Change & \% change \\
\hline BMI & $-0.38 \pm 1.8$ & $-1.4 \pm 6.7$ \\
\hline Wt & $-1 \pm 4.24$ & $-1.6 \pm 6.5$ \\
\hline P & $7.68 \pm 9.4$ & $8.2 \pm 11.3$ \\
\hline DBP & $2.5 \pm 8.3$ & $2.5 \pm 9.7$ \\
\hline SBP & $7.4 \pm 10.4$ & $5.5 \pm 7.6$ \\
\hline FPG & $3.56 \pm 13$ & $3.1 \pm 13$ \\
\hline TG & $6.9 \pm 39.97$ & $-1.22 \pm 30.44$ \\
\hline HDL-C & $-1.39 \pm 6.43$ & $-5.38 \pm 17.9$ \\
\hline LDL-C & $7.7 \pm 37.23$ & $5.27 \pm 40$ \\
\hline Tch & $7.04 \pm 19.57$ & $3.77 \pm 9.7$ \\
\hline RPP & $15.30 \pm 13.00$ & $13.45 \pm 11.6$ \\
\hline
\end{tabular}

BMI:body mass index, wt:weight, P:pulse, DBP:diastolic blood pressure, SBP:systolic blood pressure, RPP: rate pressure product, FPG: fasting plasma glucose, TG: triglycerides, HDL-C:high density lipoprotein, LDL-C: low density lipoprotein, Tch: total cholesterol

\section{DISCUSSION}

In our study, attaining a vitamin D level of 35.4 $\pm 6.8 \mathrm{ng} / \mathrm{ml}$ significantly reduced the pulse rate (PR) and the systolic blood pressure (SBP), even though no significant effect on diastolic blood pressure (DBP) was found. The reduction of PR and SBP resulted in a significant decrease in the rate pressure product $(p=<0.001)$.

In a previous study by Scragg et al, it was found that low serum $25(\mathrm{OH}) \mathrm{D}$ levels are associated with increased heart rate, systolic blood pressure, and RPP. It was also suggested that low vitamin D status may increase cardiac work in 27,153 participants aged $\geq 20$ years, in the National Health and Nutrition Examination Surveys (NHANES) carried out from 1988 to 1994 and from 2001 to $2006^{[47]}$. Our findings are completely in favor of the two reports. The significant reduction in rate pressure product in our study is an indicator of reduced cardiac work and consequently oxygen consumption as a result of raising vitamin D level. This also may partially explain the cardioprotective effect of vitamin D that was demonstrated in several studies.

In agreement with our findings, a study conducted by Pfeifer et al. In his study, 148 women aged above 70 years with serum $25(\mathrm{OH}) \mathrm{D}$ levels below $20 \mathrm{ng} / \mathrm{mL}$ were randomly administered supplements with either calcium $1200 \mathrm{mg} /$ day only or calcium $1200 \mathrm{mg} /$ day and 1,25 dihydroxycholecalciferol (DHCC) 800 IU/day for
8 weeks. Compared with calcium alone, treatment with1,25 DHCC led to a significant reduction in systolic blood pressure and heart rate, while having no significant effect on diastolic blood pressure $^{[18]}$.

A review of 11 randomized controlled vitamin D intervention trials including over 700 subjects demonstrated a reduction in the systolic (3.6 $\mathrm{mmHg})$ and diastolic $(3.1 \mathrm{mmHg})$ blood pressure at daily doses of 800-2,500 IU [ ${ }^{48]}$.

In a similar way, a second review of vitamin $D$ trials, including 2 newer studies, revealed a mean systolic blood pressure reduction of $2.44 \mathrm{mmHg}$ [49].

In a randomized, placebo-controlled study in 145 elderly women showed that 800 IU of vitamin D3 plus $1200 \mathrm{mg}$ of calcium significantly reduced blood pressure by $9.3 \%$ after 8 weeks, whereas treatment with $1200 \mathrm{mg}$ of calcium alone reduced blood pressure by only $4.0 \%(P=0.02)^{[50]}$. These findings are in consistence with ours.

Several mechanisms have been postulated to explain the blood pressure lowering effects of vitamin D. Recently, Tomaschitz et al showed that both $25(\mathrm{OH}) \mathrm{D}$ and $1,25(\mathrm{OH}) \mathrm{D}$ were inversely associated with plasma renin and angiotensin II concentrations ${ }^{[51]}$.

Other data suggested that vitamin D may play a role in regulating vascular tone by influencing the concentration of calcium in vascular smooth muscle cells (61). It may also affect the blood pressure by indirectly affecting the vascular endothelium $^{[52,53]}$.

On the other way of our results, results of a longitudinal placebo-controlled double-blind study designed by Orwoll in normotensive men who were treated with calcium and 
cholecalciferol supplements or placebo for 3 years without any demonstrable effect on systolic, diastolic, or mean arterial blood pressure ${ }^{[54]}$.

In a meta-analysis of 10 trials, the supplementation with vitamin $\mathrm{D}$ reduced systolic blood pressure non significantly, whereas there was no effect on diastolic blood pressure ${ }^{[55]}$.This is in agreement with our results regarding the absence of an effect on DBP but it differs in that it does not show a significant reduction of SBP although this was evident in our study population. Also, a short randomized double-blind trial by Scragg et al. with vitamin D supplementation at a single dose of $2.5 \mathrm{mg}$ in winter months did not show any significant decrease in blood pressure after 5 weeks when compared to placebo ${ }^{[56]}$. It is to be noted that supplementation in his study was a single high dose of vitamin D.

Studying the effect of vitamin D supplementation on blood glucose level and development of diabetes mellitus, being an important risk factor for cardiovascular diseases, has recently received great interest. Noticeably, the results of intervention studies with vitamin D supplementation on glucose homeostasis have been inconsistent ${ }^{[57,58]}$.

The effects of combined calcium and vitamin D supplementation on glucose metabolism and systemic inflammation in non-diabetic adults was reported by Pittas et al ${ }^{[59]}$. He found that daily supplementation with $500 \mathrm{mg}$ of calcium citrate and $700 \mathrm{IU}$ of vitamin $\mathrm{D}_{3}$ for three years had no apparent effect among those with normal fasting glucose although it prevented the increase in plasma glucose and insulin resistance in the subgroup of participants with impaired fasting glucose at the beginning of the study. Absence of an apparent effect on the fasting plasma glucose among adults with normal fasting plasma glucose is consistent with our findings as all of our participants had normal fasting plasma glucose at the start of the study before receiving vitamin D therapy and they did not show a significant change in FPG by the end of the study $(p=0.0184)$.

Absence of an effect of correction of vitamin D deficiency on plasma glucose levels in nondiabetic adults was also reported by Tai et al ${ }^{[25]}$.

On the other hand, in Latinos and African Americans with prediabetes and hypovitaminosis $\mathrm{D}$, doses of vitamin $\mathrm{D}$ supplementation designed to raise serum $25(\mathrm{OH}) \mathrm{D}$ levels into the uppernormal range for 1 year did not affect insulin secretion, insulin sensitivity, or the proportion of subjects who developed diabetes or whose oral glucose tolerance returned to normal ${ }^{[60]}$.
Similarly, no effect of oral vitamin D on insulin sensitivity in pre-diabetes patients in 12 weeks treatment, in a study conducted by Seyed et al ${ }^{[61]}$. Although many cross sectional studies reported an inverse correlation between vitamin D3 level and BMI, results of interventional studies are controversial.

The data on vitamin $\mathrm{D}$ supplementation during weight loss were too few to make firm conclusions. Current evidence from randomized controlled trials did not consistently support the contention that calcium and vitamin D accelerated weight or fat loss in obesity. There were studies that favoured the hypothesis but lacked the statistical power. There is a need for RCTs to examine the influence of vitamin D on body fat [62]

In our study we did not find a significant effect of vitamin D supplementation on the BMI. Although we did not measure the body fat mass in our study, it may be in consistence with results of a study conducted by Saleh et al. Saleh and his colleagues studied the impact of a 12 week vitamin D3 supplementation on anthropometric indices in healthy overweight and obese women. Supplementation caused statistically significant decrease in body fat mass in the vitamin D group compared to the placebo group. However body weight and consequently BMI as well as waist circumference did not significantly change ${ }^{[63]}$.

We studied the different components of lipid profile before and after replenishment of vitamin D. Vitamin D did not significantly affect the total cholesterol, LDL-C, HDL-C or triglyceride level. This is in contrast to the cross-sectional association between 25(OH)D levels and a healthier lipid profile.

Jorde et al, reported findings similar to ours. He found that raising $25(\mathrm{OH}) \mathrm{D}$ levels from deficient to optimal in a cohort neither improved nor worsened the lipid profile. This suggests that a higher level of $25(\mathrm{OH}) \mathrm{D}$ may simply be a passive marker of better cardiovascular health. People in good health stay outdoors more and therefore get more sunshine and vitamin $\mathrm{D}$ production in the skin, and they may also have healthier food habits. Their higher serum 25(OH)D levels may therefore be the result and not the cause of good health ${ }^{[64]}$. Similarly, Ponda et al found small and clinically minimal effects on total and HDL cholesterol with no benefit on LDL cholesterol and triglycerides in 51 vitamin D-deficient adults who received 50 000 international units of vitamin D weekly for 8 weeks. Ponda and colleagues suggest that vitamin D status is a surrogate marker of health and that vitamin D might simply have no effect on lipid 
metabolism. Moreover, they see that dyslipidemia might be responsible for the low vitamin D levels and not the other way around ${ }^{[65]}$

In agreement with our findings, results reported by Guri et al. In his study, participants with low serum 25(OH)D levels were randomized to receive capsules of 20,000 IU vitamin $D_{3}$ or identical-looking placebo twice weekly for 6 months. At the end of the study, there were no statistically significant differences in the outcome variables including LDL-C, HDL-C and triglycerides between the two groups ${ }^{[66]}$.

Hao et al conducted a meta analysis, the results of which had a great agreement with our report. In all, 12 clinical trials consisting of 1346 participants, no statistically significant effects for vitamin $\mathrm{D}$ supplementation were observed for total cholesterol, HDL-C and triglycerides ${ }^{[67]}$.

Results of the forementioned studies and our study indicate that vitamin D supplementation to apparently healthy vitamin $\mathrm{D}$ deficient adults does not improve serum lipid profile.

Absence of an effect of vitamin D supplementation on lipid profile despite the association of vitamin D deficiency with the prevalence of traditional CVD risk factors, suggests a more direct role of vitamin $\mathrm{D}^{[68]}$.

Of course associations do not prove causation; they can only generate hypotheses. Different ethnicity, age group, anthropometric parameters, given dose of vitamin $\mathrm{D}$, target vitamin $\mathrm{D}$ level which was achieved, duration of the study, and state of glucose homeostasis before starting the treatment of deficiency, all of which may have a role in such controverse in the outcome of several studies.

In a trial to find out the optimal level of vitamin D that can have beneficial effect on CVD, a recent review of randomized trials evaluating the effect of greater doses and higher achieved 25dihydroxyvitamin D [25(OH)D] levels on health outcomes concluded that the optimal benefit of vitamin D for CVD was obtained at $25(\mathrm{OH}) \mathrm{D}$ concentrations of 30-44 ng/ml. These levels were possible to achieve with oral daily doses of vitamin D in the range of 1,800 to $4,000 \mathrm{IU}^{[5]}$.

\section{CONCLUSIONS}

From the current study we may conclude that in South West Asian population, vitamin D replenishment in vitamin $\mathrm{D}$ deficient healthy adults does not lead to a significant reduction of the fasting plasma glucose or a significant change in the lipid profile.

On the other hand, vitamin D replacement leads to a significant reduction in the systolic blood pressure, the pulse rate as well as the rate pressure product. Reduction of the RPP indicates a reduction of the cardiac work and consequently the oxygen consumption by the heart. This may partially explain the cardioprotective effect of vitamin D therapy.

\section{REFERENCES}

1-Lopez AD, Mathers CD, Ezzati M, Jamison DT, Murray CJ: Global and regional burden of disease and risk factors: systematic analysis of population health data. Lancet 2006; 367:17471757.

2-Global Atlas on Cardioavascular Disease Prevention and Control. Mendis S, Puska P,Norrving B editors. World Health Organization (in collaboration with the World Heart Fedration and World Stroke Organization), Geneva 2011.

3-Chaoxun Wang. Role of Vitamin D in Cardiometabolic Diseases. J Diabetes Res. 2013;2013:243934. doi: 10.1155/2013/243934. PMID:23671861 PMCID: PMC3647592

4-Holick MF: Vitamin D deficiency. $N$ Engl $J$ Med 2007, 357: 266-281.

5-Olivera Ilić Stojanovic, Milica Lazovic, Marko Lazovic, Marina Vuceljic :Association between atherosclerosis and osteoporosis, the role of vitamin D. Arch Med Sci 2011; 7: 17918. DOI: $10.5114 /$ aoms.2011.22066

6-David Martins, Myles Wolf, Deyu Pan, Ashraf Zadshir, Naureen Tareen et al: Prevalence of Cardiovascular Risk Factors and the Serum Levels of 25-Hydroxyvitamin D in the United States Data From the Third National Health and Nutrition Examination Survey. Arch Intern Med. $\quad$ 2007;167:1159-1165. doi:10.1001/archinte.167.11.1159

7-Zittermann A: Vitamin D and disease prevention with special reference to cardiovascular disease. Prog Biophys Mol Biol 2006; 92: 39-48.

8-Forman JP, Williams JS, Fisher ND: Plasma 25hydroxyvitamin $\mathrm{D}$ and regulation of the reninangiotensin system in humans. Hypertension 2010; 55:1283-1288.

9-Kendrick J, Targher G, Smits G, Chonchol M: Hydroxyvitamin D deficiency is independently associated with cardiovascular disease in the Third National Health and Nutrition Examination Survey. Atherosclerosis 2009;205:255-60. doi: 10.1016/j.atherosclerosis.2008.10.033. PMID:19091317

10-Hintzpeter B, Mensink GB, Thierfelder W, Muller MJ, Scheidt-Nave C. Vitamin D status and health correlates among German adults. Eur J Clin Nutr. 2008; 62: 1079-1089. 
11-Hypponen E, Boucher BJ, Berry DJ, Power C. 25-Hydroxyvitamin D, IGF-1, and metabolic syndrome at 45 years of age: a cross-sectional study in the 1958 British Birth Cohort. Diabetes. 2008; 57: 298-305

12-Liu E, Meigs JB, Pittas AG, Mc Keown NM, Economos CD, Booth SL, Jacques PF: Plasma 25-hydroxyvitamin $\mathrm{d}$ is associated with markers of the insulin resistant phenotype in non-diabetic adults. J Nutr. 2009; 139 (2): 32934 doi: 10.3945/jn.108.093831.

13-Pittas AG, Dawson-Hughes B: VitaminD and diabetes. J Steroid Biochem Mol Biol 2010;121:425-9. doi: 10.1016/j.jsbmb.2010.03.042. Epub 2010 Mar 18. PMID:20304061 PMCID:PMC2900448

14-Voidonikola PT, Stamatelopoulos KS, Alevizaki M, Kollias GE, Zakopoulos NA et al: The association between glycemia and endothelial function in nondiabetic individuals: the importance of body weight. Obesity (Silver Spring) 2008; 16:2658-62. doi: 10.1038/oby.2008.431.

15-Liu E, Meigs JB, Pittas AG, Economos CD, McKeown NM et al: Predicted 25hydroxyvitamin D score and incident type 2 diabetes in the Framingham Offspring Study. Am J Clin Nutr. 2010; 91:1627-33. doi: 10.3945/ajcn.2009.28441. PMID:20392893

16-Forouhi NG, Ye Z, Rickard AP, Khaw KT, Luben $\mathrm{R}$ et al: Circulating 25-hydroxyvitamin $\mathrm{D}$ concentration and the risk of type 2 diabetes: results from the European Prospective Investigation into Cancer (EPIC)-Norfolk cohort and updated meta-analysis of prospective studies. doi:10.1186/1475-284011-85

17-Mitri J, Muraru MD, Pittas AG: Vitamin D and type 2 diabetes: a systematic review. Eur J Clin Nutr. 2011; 65:1005-15. doi: 10.1038/ejcn.2011.118.

18- Pittas AG, Chung M, Trikalinos T, Mitri J, Brendel $\mathrm{M}$ et al: Systematic review: Vitamin D and cardiometabolic outcomes. Ann Intern Med 2010, 152:307-314. doi: 10.7326/00034819-152-5-201003020-00009.

19-Ravi K Menon, Anna P Rickard, Nasima Mannan, Peter M Timms, Stephen J Sharp et al: The effects of vitamin $D_{2}$ or $D_{3}$ supplementation on glycaemic control and related metabolic parameters in people at risk of type 2 diabetes: protocol of a randomised double-blind placebo-controlled trial. $B M C$ Public Health 2013;13:999 PMID:24152375 PMCID:PMC3819003
20-Liu E, McKeown NM, Newby PK, Meigs JB, Vasan RS et al: Cross-sectional association of dietary patterns with insulin-resistant phenotypes among adults without diabetes in the Framingham Offspring Study. $\mathrm{Br} J \mathrm{Nutr}$. 2009;102:576-83. doi: 10.1017/S0007114509220836.PMID: 1921682 8

21-Yoshida M, Booth SL, Meigs JB, Saltzman E, Jacques PF: Phylloquinone intake, insulin sensitivity, and glycemic status in men and women. Am J Clin Nutr 2008; 88:2105.PMID:18614743

22-Rumawas ME, Meigs JB, Dwyer JT, McKeown NM, Jacques PF: Mediterraneanstyle dietary pattern, reduced risk of metabolic syndrome traits, and incidence in the Framingham Offspring Cohort. Am J Clin Nutr 2009; 90:1608-14. doi: 10.3945/ajcn.2009.27908. PMID: 19828705

23-Smotkin-Tangorra M, Purushothaman R, Gupta A, Nejati G, Anhalt H et al: Prevalence of vitamin $\mathrm{D}$ insufficiency in obese children and adolescents. Journal of Pediatric Endocrinology and Metabolism 2007; 20: 817 823.

24-Scragg R, Sowers M \& Bell C: Serum 25hydroxyvitamin D, ethnicity, and blood pressure in the Third National Health and Nutrition Examination Survey. American Journal of Hypertension 2007; 20: 713-719.

25-Tai K, Need AG, Horowitz M, Chapman IM: Vitamin D, glucose, insulin, and insulin sensitivity. Nutrition 2008; 24: 279-285.

26-Need AG, O'Loughlin PD, Horowitz M, Nordin BE: Relationship between fasting serum glucose, age, body mass index and serum 25 hydroxyvitamin $\mathrm{D}$ in postmenopausal women. Clinical Endocrinology 2005; 62: 738-741.

27- de Boer IH, Tinker LF, Connelly S, Curb JD, Howard BV: Women's Health Initiative Investigators. Calcium plus vitamin D supplementation and the risk of incident diabetes in the Women's Health Initiative. Diabetes Care 2008; 31: 701-707.

28-Pittas AG, Harris SS, Stark PC, DawsonHughes B: The effects of calcium and vitamin D supplementation on blood glucose and markers of inflammation in nondiabetic adults. Diabetes Care 2007; 30: 980-986.

29-Botella-Carretero JI, Alvarez-Blasco F, Villafruela JJ, Balsa JA, Vazquez C, EscobarMorreale HF: Vitamin D deficiency is associated with the metabolic syndrome in 
morbid obesity. Clin Nutr 2007; 26 (5): 57380.

30-Florez H, Martinez R, Chacra W, StrickmanStein N, Levis S: Outdoor exercise reduces the risk of hypovitaminosis $\mathrm{D}$ in the obese. $\mathrm{J}$ Steroid Biochem. Mol. Biol., 103: 679-681, 2007.

31-Jorde R, Grimnes G: Vitamin D and metabolic health with special reference to the effect of vitamin D on serum lipids. Prog Lipid Res 2011; 50:303-12. doi: 10.1016/j.plipres.2011.05.001.

32- Bell DS: Protean manifestations of vitamin D deficiency, part 3: association with cardiovascular disease and disorders of the central and peripheral nervous systems.

South Med J 2011;104:340-4. doi: 10.1097/01.SMJ.0000397894.02150.5f.

33-Sambrook PN, Chen CJ, March L et al: High bone turnover is an independent predictor of mortality in the frail elderly. J Bone Miner Res 2006; 21: 549-55.

34- Holick MF: High prevalence of vitamin D inadequacy and implications for health. Mayo Clin Proc 2006; 81:353-73. PMID:16529140

35- Boonen S, Bischoff-Ferrari HA, Cooper C, Lips $\mathrm{P}$, Ljunggren $\mathrm{O}$ et al: Addressing the musculoskeletal components of fracture risk with calcium and vitamin D: a review of the evidence. Calcif Tissue Int. 2006;78:257-70. PMID:16622587.

36-Bakhtiyarova S, Lesnyak O, Kyznesova N, Blankenstein MA, Lips P: Vitamin D status among patients with hip fracture and elderly control subjects in Yekaterinburg, Russia. Osteoporos Int. 2006;17:441-6. PMID: 16328605

37-Lips P, Hosking D, Lippuner K, Norquist JM, Wehren $L$ et al: The prevalence of vitamin D inade-quacy amongst women with osteoporosis: an international epidemiological investigation. J Intern Med 2007; 261:408. PMID: 16918822

38- Mithal A, Wahl DA, Bonjour JP, Burckhardt P, Dawson-Hughes Betl: Global vitamin D status and determinants of hypovitaminosis D. Osteoporos Int 2009; 20:1807-1820.

39-Lee JH, O'Keefe JH, Bell D, Hensrud DD, Holick MF: Vitamin D deficiency an important, common, and easily treatable cardiovascular risk factor? J Am Coll Cardiol 2008; 52:1949-1956.

40-Hao Wang, Ning Xia, Yang Yang, Dao-Quan Peng: Influence of vitamin $D$ supplementation on plasma lipid profiles: A meta-analysis of randomized controlled trials. Lipids Health
Dis. 2012; 11: 42. doi: 10.1186/1476-511X11-42 PMCID: PMC3325888

41- Chaney RH: Comparison of blood pressure and rate-pressure product responses in maximal dynamic and isometric exercise. Ann Acad Med Singapore 1981;10:7-10

42-Vogeser M, Apostolos Kyriat Soulis: Candidate Reference. Methodology for the Quantification of Circulating 25-Hydroxy vitamin D3 by Liquid ChromatographyTandem Mass Spectrometry. Clin Chem 2004; 50: 1415-1517.

43-Fossati P, Prencip L: Serum triglycerides determined colorimetrically with an enzyme that produces hydrogen peroxidase. Clin Chem 1982; 2077-2080.

44- Hino k, Nakamura $M$ et al: Anew method for the homogenous assay of serum HDLcholesterol. Clin Chem 1996; 42:5299.

45-Trinder P: Determination of glucose in blood using glucosenoxidase with an alternative oxygen acceptor. Ann Clin Biochemis 1969; 6: 24-27.

46-Hollis BW, Wagner CL. Normal serum vitamin $\mathrm{D}$ levels. $\mathrm{N}$ Engl $\mathrm{J}$ Med. Feb 3 2005;352(5):515-6.

47-Lambert M, Sumukadas D, Witham M: Serum phosphate in older people. Reviews in Clinical Gerontology 2009; 4: 263-270doi: 10.1017/S0959259809990359

48- Witham MD, Nadir MA, Struthers AD. Effect of vitamin D on blood pressure: a systematic review and meta-analysis. J. Hypertens. 2009 Oct.;27(10):1948-1954.

49-Wu SH, Ho SC, Zhong L: Effects of vitamin D supplementation on blood pressure. South Med J 2010; 8:729-37. doi: 10.1097/SMJ.0b013e3181e6d389.

50-M. Pfeifer, B. Begerow, H. W. Minne, D. Nachtigall, and C. Hansen, "Effects of a shortterm vitamin D3 and calcium supplementation on blood pressure and parathyroid hormone levels in elderly women," The Journal of Clinical Endocrinology \& Metabolism, vol. 86, no. 4, pp. 1633-1637, 2001.

51-Tomaschitz A, Pilz S, Ritz E, Grammer T, Drechsler C, Boehm BO, Marz W: Independent association between 1,25dihydroxyvitamin D, 25-hydroxyvitamin D and the renin-angiotensin system: the Ludwigshafen Risk and Cardiovascular Health (LURIC) Study. Clin Chim Acta 2010; 411: 1354-1360.

52-Shan J, Resnick LM, Lewanczuk RZ, Karpinski E, Li B, Pang PK: 1,25Dihydroxyvitamin $\mathrm{D}$ as a cardiovascular 
hormone: effects on calcium current and cytosolic free calcium in vascular smooth muscle cells. Am J Hypertens. 1993; 6: 983988.

53-Tarcin O, Yavuz DG, Ozben B, Telli A, Ogunc AV et al: Effect of vitamin D deficiency and replacement on endothelial function in asymptomatic subjects. J Clin Endocrinol Metab. 2009; 94: 4023-4030.

54-Orwoll ES, Oviatt S: Relationship of mineral metabolism and long-term calcium and cholecalciferol supplementation to blood pressure in normotensive men. The American Journal of Clinical Nutrition 1990; 4:717-721.

55- Pfeifer $M$, Begerow $B$, Minne $H$ W, Nachtigall D, Hansen C: Effects of a shortterm vitamin D3 and calcium supplementation on blood pressure and parathyroid hormone levels in elderly women. Journal of Clinical Endocrinology and Metabolism 2001; 4: 1633-1637.

56- Scragg R, Khaw K-T, Murphy S: Effect of winter oral vitamin D3 supplementation on cardiovascular risk factors in elderly adults. European Journal of Clinical Nutrition 1995; 9:640-646.

57-Borissova AM, Tankova T, Kirilov G, Dakovska L, Kovacheva R: The effect of vitamin D3 on insulin secretion and peripheral insulin sensitivity in type 2 diabetic patients. Int J Clin Pract 2003; 57:258-261.

58-Zemel MB, Thompson W, Milstead A, Morris K, Campbell P: Calcium and dairy acceleration of weight and fat loss during energy restriction in obese adults. Obes Res 2004; 12:582-590.

59-Pittas-Anastassos G, Susan S Harris, Paul C. Stark, Bess Dawson-Hyghes: The Effects of Calcium and Vitamin D Supplementation on Blood Glucose and Markers of Inflammation in Non-diabetic Adults. Diabetes Care 2007; doi: $10.2337 / \mathrm{dc} 06-1994$

60-Mayer B, Petra D, Martin L, Theodore C: High-Dose Vitamin D Supplementation in People with Prediabetes and Hypovitaminosis D. Diabetes Care 2013; 2: 260-266
61- Seyed Hoseini, Ashraf Aminorroaya, Bijan Iraj, and Massoud Amini: The effects of oral vitamin $\mathrm{D}$ on insulin resistance in pre-diabetic patients. J Res Med Sci 2013; 18: 47-51. PMCID: PMC3719226.

62-Soares MJ, Chan She Ping-Delfos W, Ghanbari MH.Calcium and vitamin D for obesity: a review of randomized controlled trials. Eur J Clin Nutr. 2011 Sep;65(9):9941004. doi: 10.1038/ejen.2011.106.

63-Salehpour A, Hosseinpanah F, Shidfar F, Vafa M, Razaghi M, Dehghani S, Hoshiarrad A: A 12-week double-blind randomized clinical trial of vitamin D3 supplementation on body fat mass in healthy overweight and obese women. Nutr J. 2012 ; 11(1):78.

64- Jorde R, Grimnes G: Vitamin D and lipids: do we really need more studies. Circulation 2012;126:252-4. doi: 10.1161/CIRCULATIONAHA.112.119693.

65- Ponda MP, Huang X, Odeh MA, Breslow JL, Kaufman HW: Vitamin D may not improve lipid levels: a serial clinical laboratory data study. Circulation 2012;126:270-7. doi: 10.1161/CIRCULATIONAHA.111.077875.

66-Guri Grimnes, Yngve Figenschau, Bjørg Almås, Rolf Jorde: Vitamin D, Insulin Secretion, Sensitivity, and Lipids: Results From a Case-Control Study and a Randomized Controlled Trial Using Hyperglycemic Clamp Technique. Diabetes 2011; 60: 2748-2757. doi: $\quad 10.2337 / \mathrm{db} 11-0650 \quad$ PMCID: PMC3198082

67- Wang H, Xia N, Yang Y, Peng DQ: Influence of vitamin $\mathrm{D}$ supplementation on plasma lipid profiles: a meta-analysis of randomized controlled trials. Lipids Health Dis 2012; 11:42. doi: 10.1186/1476-511X-11-42.

68-Anagnostis P, Athyros VG, Adamidou F, Florentin M, Karagiannis A: Vitamin D and cardiovascular disease: a novel agent for reducing cardiovascular risk? Curr Vasc Pharmacol 2010; 8: 720-30. 